\title{
DONOR LYMPHOCYTE INFUSION FOR LATE RELAPSE FOLLOWED BY KIDNEY TRANSPLANTATION FOR END-STAGE RENAL FAILURE AFTER ALLOGENEIC BONE MARROW TRANSPLANTATION FOR CHRONIC MYELOID LEUKEMIA
}

A 36-year-old man was diagnosed in 1986 with chronic phase (CP) chronic myeloid leukemia (CML). Three months later, he developed a blast crisis and blastic embolization of his left tibial artery requiring a left foot amputation. After successful induction and consolidation therapy, the patient (then in second CP) underwent an allogeneic bone marrow transplantation (BMT) from his human leukocyte antigen-identical brother. The conditioning regimen consisted of cyclophosphamide, cytarabine, and 8-Gy total body irradiation, and graft-versus-host disease (GVHD) prophylaxis was carried out with cyclosporine A alone. The immediate posttransplant course was complicated by grade 3 GVHD that was successfully treated with steroids and methotrexate, and the patient was discharged on day 43. At that time, his serum creatinine was $16 \mathrm{mg} / \mathrm{L}$ (Fig. 1). Limited chronic GVHD was diagnosed on day 100 and was successfully treated with psoralen and long-wave ultraviolet radiation. Thereafter, the evolution remained uneventful except for hypertension. Although cyclosporine A was discontinued on day 420, his renal function progressively degraded (Fig. 1). In 1994, the patient's serum creatinine was $32 \mathrm{mg} / \mathrm{L}$ and a renal biopsy revealed tubulointerstitial nephritis with nephrosclerosis. Gradual deterioration of his renal function resulted in the institution of maintenance hemodialysis in August 1996. A kidney transplant from his marrow donor brother was considered in March 1998. However, the patient's bone marrow analysis at that time showed a cytogenetic relapse (4 of 26 phi-positive metaphases) and mixed chimerism. He thus received donor lymphocyte infusion (DLI) $\left(1 \times 10^{7} \mathrm{CD}^{+}\right.$cells/kg). The patient did not experience acute or chronic $\mathrm{GVHD}$, and bone marrow analyses 3 and 7 months later showed a complete cytogenetic and molecular response. Chimerism analysis in December 1999 demonstrated full donor chimerism.

After his brother, who had given bone marrow to him 14 years earlier, was found to be an acceptable donor, kidney transplantation was performed in April 2000, without any immunosuppressive therapy. The transplanted kidney functioned immediately, and a kidney biopsy performed 6 months after the transplant did not show any sign of graft rejection (Fig. 1). Now, more than 2 years after kidney transplantation, the patient is well with normal renal function and without any sign of GVHD or CML.

Although late-onset renal failure occurs in up to $20 \%$ of survivors of allogeneic bone marrow transplantation, chronic renal failure requiring dialysis is a relatively rare event in the BMT setting (approximately $2 \%$ of the patients) (1). The feasibility of kidney transplantation after allogeneic BMT has already been reported (2-4). However, our report is unique in that the patient was in relapse when the kidney transplant procedure was contemplated but could be rescued by a DLI.

It is now well established that DLI can cure CML in patients relapsing after an allogeneic transplant (5). Because leukemic relapse may be accompanied by residual host hematopoiesis that would imply a requirement for restoration of immunosuppressive therapy to avoid kidney graft rejection and thereby alter the DLI-mediated graft-versus-leukemia effect, we decided to delay kidney transplantation until well after DLI and achievement of full donor chimerism. In conclusion, our report emphasizes that renal transplantation should not be ruled out in patients with DLI-sensitive disease relapsing after an allogeneic BMT.
FIGURE 1. Evolution of serum creatinine (continuous line) and creatinine clearance (broken line) throughout the posttransplant course.

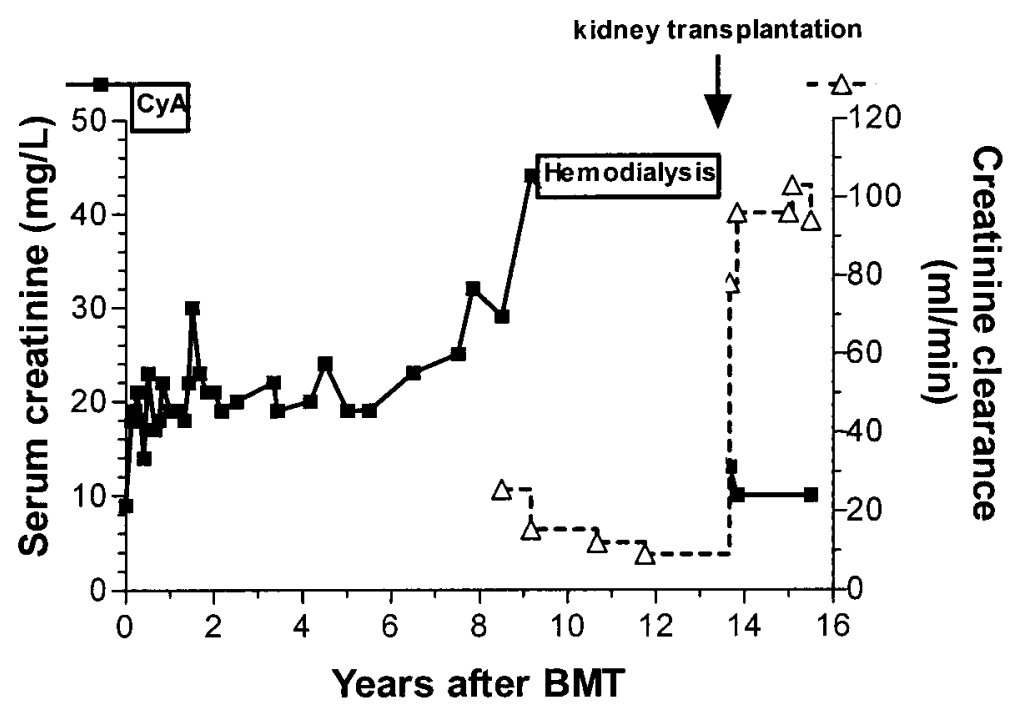


StÉPHANie Humblet-BaroN

FRÉDÉRIC BARON

Yves Beguin

Department of Medicine

Division of Hematology

University of Liège

Liège, Belgium

ALFRED Chachati

Department of Medicine

Division of Nephrology

Centre Hospitalier Hutois

Huy, Belgium

JEAN BURY

Department of Medicine

Division of Hemato-Oncology

Centre Hospitalier Hutois

Huy, Belgium

EMMANUEL MORELON

HENRI KREIS

Department of Renal Transplantation

Necker Hospital

Paris, France

DOI: 10.1097/01.TP.0000085285.59061.D7
Frédéric Baron is Research Assistant and Yves Beguin Research Director of the National Fund for Scientific Research, Belgium.

This work was supported in part by grants from the National Fund for Scientific Research.

Address correspondence to: Yves Beguin, M.D., Department of Hematology, University of Liège, CHU Sart-Tilman, 4000 Liège, Belgium. E-mail: yves.beguin@chu.ulg.ac.be

Received 6 May 2003. Accepted 14 may 2003.

\section{MIXED CHIMERISM OF THYROID FOLLICLE CELLS AFTER ALLOGENEIC BONE MARROW TRANSPLANTATION}

Recently, a growing amount of data have been accumulated concerning the plasticity of human adult stem cells that may generate a multilineage engraftment. In this context, there is some evidence of a putative relationship between mixed microchimerism in thyroid follicle cells (1) of presumed fetal origin and autoimmune disease in female patients $(2-4)$. A model to study this issue would include patients in whom bone marrow transplantations (BMTs) with a sex-mismatched constellation were performed. Therefore, a retrospective analysis of thyroid gland tissue derived from four male patients with chronic myelogenous leukemia who underwent allogeneic BMTs was performed. These patients received full unmanipulated grafts (median size $2.3 \times 10^{8} / \mathrm{kg}$ nucleated cells) from sex-mismatched human leukocyte antigen-identical (female) family donors following standard procedures that included conditioning regimens $(\mathrm{Cy} 60 \mathrm{mg} / \mathrm{kg} /$ day $\times 2$; dose of total body irradiation $4 \times 2.5$ Gy 60 cobalt) and graftversus-host-disease prophylaxis (short-term methotrexate + cyclosporine A). Leukemic relapse occurred in two patients after a posttransplant observation time ranging from 2 to 26 months. Relevant grades of acute graft-versus-host-disease accompanied by lethal infections developed in the other two patients of this series approximately 3 to 12 weeks after BMT. As controls, postmortem tissue samples without evidence of thyroid pathology were obtained from two male and two female patients.

During the autopsy, samples of thyroid gland tissue were randomly taken from the right and left lobes and fixed in buffered 5\% formalin. For dual-color genotypic evaluation, paraffin-embedded sections were used and processed by a previously described modified fluorescence in situ hybridization (FISH) technique applying $\mathrm{x}$ - and $\mathrm{y}$ chromosome probes (5). To neutralize possible artefacts in the autopsy material and to match with the obstacles of a certain plane of section regarding proper signal recognition, only cells were evaluated that displayed two distinctive red and green dots.

After FISH, distinctively labeled thyroid epithelial cells lining the walls of the colloid-containing follicles were easily identifiable. In each of the four patients of the control group, 300 follicle cells were counted; however, FISH failed to reveal any sex-mismatched chromosome signals in this cohort. Contrasting this finding in the four patients with a history of BMT, a striking, although quantitatively low, number of thyroid epithelium displayed mixed microchimerism (Table 1). It is noteworthy that this small amount of female donor cells among the host-type (male) thyroid follicle cells was not related to the period of engraftment.

Considering the negative findings in the control group of patients, sensitivity of the FISH technique seems to be

TABLE 1. Mixed chimerism of the thyroid follicle cells in a sex-mismatched (host male, donor female) allogeneic bone marrow transplantation constellation (percentages are listed in brackets)

\begin{tabular}{lccc}
\hline Patient & $\begin{array}{c}\text { Number of } \\
\text { follicle cells }\end{array}$ & $\begin{array}{c}\text { yx (green/red) } \\
\text { signals }\end{array}$ & $\begin{array}{c}\text { xx (red/red) } \\
\text { signals }\end{array}$ \\
\hline 1 & 354 & 331 & $23(6.5)$ \\
2 & 387 & 369 & $18(4.7)$ \\
3 & 349 & 331 & $18(5.2)$ \\
4 & 626 & 606 & $20(3.2)$ \\
Total & 1,716 & 1,637 & $79(4.6)$ \\
\hline
\end{tabular}

